\title{
Turbulence And Coherent Structures in Two-Component Bose Condensates
}

\author{
Natalia G. Berloff and Chen Yin \\ Department of Applied Mathematics and Theoretical Physics, \\ University of Cambridge, Cambridge, CB3 0WA
}

We elucidate the self-evolution of two Bose gases from a strongly non equilibrium initial state. Large scale numerical simulations of the coupled nonlinear Schrödinger equations are used to follow the evolution of the system from weak turbulence to strong turbulence to superfluid turbulence in the long-wavelength region of energy space with a formation of a tangle of topological defects. The addition of the second gas increases the number of condensed particles in the first gas. It is shown that the large wavelength part of the fields evolves into coherent structures identified as solitary wave complexes of the coupled nonlinear Schrödinger equations. The families of the solitary waves moving on uniform background or along the topological defects are obtained as solutions of the coupled Gross-Pitaevskii equations. It is shown that there exist three continuous families of such solutions with or without the cusp in energy-momentum space.

PACS numbers: 03.75.Fi, 02.60.Cb, 05.45.-a, 47.20.Ky

\section{INTRODUCTION}

The systems of the coupled nonlinear Schrödinger (NLS) equations are fundamental and universal systems that have been used to describe motions in conservative systems of weakly nonlinear dispersive waves in continuum mechanics, plasma physics, nonlinear optics and condensed matter. The coupled NLS equations have been receiving a lot of attention with recent experimental advances in multi-component Bose-Einstein condensates (BECs) formed by simultaneous trapping and cooling of atoms in distinct spin or hyperfine levels ${ }^{1}$ or of different atomic species ${ }^{2}$. There is also an interest in the condensation and dynamics of multi-component BECs within the COSLAB framework: multi-component BECs offer the simplest tractable 


\section{Natalia G. Berloff and Chen Yin}

microscopic models in the proper universality class of cosmological systems 3 .

When a weakly interacting Bose gas is so rapidly cooled below the critical BEC temperature that the particles remain in a strongly nonequilibrium state, an accurate microscopic description of the BEC formation is given by the NLS equation ${ }^{4}$. The kinetics of the initial weak turbulent state has been analysed in detail ${ }^{5-7}$ revealing a quasi-particle cascade from high energies to low energies in the wave number space. The regime of weak turbulence breaks down in a low-energy region when the superfluid short-range order sets in leading to a state of superfluid turbulence with a tangle of quantised vortices. The transition between the different regimes was elucidated using large scale numerical simulations of the NLS equation in $3 \mathrm{D}^{8,9}$ and $2 \mathrm{D}{ }^{10}$.

The system is initially in the weak turbulence regime and is described by the Boltzmann kinetic equation. The system evolves in a self-similar fashion in which the quasi-particles cascade to lower and lower energies until the assumption of a random-phase approximation breaks down and the system enters the coherent regime (strong turbulence). In this regime the phases of the complex Fourier amplitudes become strongly correlated, the periods of their oscillations and the evolution times of the occupation numbers become comparable. During this regime the quasi-condensate is formed with a tangle of well-defined quantised vortices. The tangle relaxes during superfluid turbulence regime which is the final stage of the field evolution which has been studied in detail ${ }^{11}$.

In this paper we consider the condensation of two Bose gases. In the limit of weak interactions and strong nonequilibrium initial condition, the key stage of ordering dynamics (the formation of superfluid turbulence) is universal and corresponds to the process of self-ordering of classical matter fields according to the evolution of the coupled NLS equations.

When two weakly interacting Bose gases are simultaneously rapidly cooled below the transition temperature their evolution is described by the coupled NLS equations in analogy with a single component Bose gas

$$
\begin{aligned}
i \hbar \frac{\partial \psi_{1}}{\partial t} & =\left[-\frac{\hbar^{2}}{2 m_{1}} \nabla^{2}+V_{11}\left|\psi_{1}\right|^{2}+V_{12}\left|\psi_{2}\right|^{2}\right] \psi_{1}, \\
i \hbar \frac{\partial \psi_{2}}{\partial t} & =\left[-\frac{\hbar^{2}}{2 m_{2}} \nabla^{2}+V_{12}\left|\psi_{1}\right|^{2}+V_{22}\left|\psi_{2}\right|^{2}\right] \psi_{2},
\end{aligned}
$$

where $\psi_{1}$ and $\psi_{2}$ are complex-valued classical fields that specify the indices of the coherent states of two gases, $m_{i}$ is the mass of the atom of the $i$ th gas, and the coupling constants $V_{i j}$ are proportional to scattering lengths $a_{i j}$ via $V_{i j}=2 \pi \hbar^{2} a_{i j} / m_{i j}$, where $m_{i j}=m_{i} m_{j} /\left(m_{i}+m_{j}\right)$ is the reduced mass. The 


\section{Two-Component Bose Condensates}

energy functional of the system (e.g. [12]) is

$$
E=\int\left[\sum_{i=1}^{2}\left\{\frac{\hbar^{2}}{2 m_{i}}\left|\nabla \psi_{i}\right|^{2}+\frac{1}{2} V_{i i}\left|\psi_{i}\right|^{4}\right\}+\left|\psi_{1}\right|^{2} V_{12}\left|\psi_{2}\right|^{2}\right] d V .
$$

We assume that upon a fast cooling there arises an isolated system that consists of $N_{1}$ particles of one Bose gas and $N_{2}$ particles of another Bose gas with the energy per particle much less than the critical BEC temperature that corresponds to the given particle densities. Without loss of generality we shall also assume that $N_{2} \leq N_{1}$.

We are interested in the evolution of two strongly nonequilibrium fields:

$$
\psi_{1}=\sum_{\mathbf{k}} a_{\mathbf{k}} \exp (\mathrm{ik} \cdot \mathbf{r}), \quad \psi_{2}=\sum_{\mathbf{k}} b_{\mathbf{k}} \exp (\mathrm{ik} \cdot \mathbf{r})
$$

where the phases of the complex amplitudes $a_{\mathbf{k}}$ and $b_{\mathbf{k}}$ are distributed randomly.

In this paper we consider two questions (1) what effect does the addition of the second component has on the process of condensation? and (2) what coherent structures are formed during the condensation? We shall show that the presence of the second gas dramatically increases the number of condensed particles in the first gas if the equilibrium temperature of the second gas alone is lower than that of the first gas. This increase is maximal when $N_{2} / N_{1} \approx 1 / 2$. The number of condensed particles in the second gas is lowered in comparison with the condensation of this gas alone.

The paper is organized as follows. In Section 2 we derive the kinetic equation for the evolution of occupation numbers of two components and analyze the wave spectrum. Large-scale numerical simulations are used to show the formation of condensates with a tangle of topological defects. In Section 3 we study the families of solitary wave solutions to which the condensates evolve in the process of turbulence decay. Solitary waves moving along the topological defects are found in Section 4. We conclude with a summary of our findings in Section 5 .

\section{CONDENSATION IN A COUPLED GROSS-PITAEVSKII MODEL}

In this section we consider the problem of self-organization in a coupled NLS model (1) starting with random initial conditions (3). For such a random field we can derive an irreversible kinetic equation for the averaged wave spectrum. 


\section{Natalia G. Berloff and Chen Yin}

The NLS equations (1) written for the Fourier components $a_{\mathbf{k}}$ and $b_{\mathbf{k}}$ take the form

$$
\begin{aligned}
& i \dot{a}_{1}=\epsilon_{1} a_{1}+\sum_{234}\left(V_{11} a_{2}^{*} a_{3} a_{4}+V_{12} b_{2}^{*} b_{3} a_{4}\right) \delta_{1 ; 3+4-2}, \\
& i \dot{b}_{1}=\epsilon_{2} b_{1}+\sum_{234}\left(V_{12} a_{2}^{*} a_{3} b_{4}+V_{22} b_{2}^{*} b_{3} b_{4}\right) \delta_{1 ; 3+4-2},
\end{aligned}
$$

where $\epsilon_{i}=k_{i} / 2 m_{i}, \hbar=1$ and the notation $\mathbf{k}_{i} \rightarrow i$ was used, so that for instance, $\delta_{1 ; 3+4-2}=\delta\left(\mathbf{k}_{1}+\mathbf{k}_{2}-\mathbf{k}_{3}-\mathbf{k}_{4}\right)$. The occupation numbers $n_{\mathbf{k}}=\left|a_{\mathbf{k}}\right|^{2}$ and $l_{\mathbf{k}}=\left|b_{\mathbf{k}}\right|^{2}$ obey

$$
\begin{aligned}
& \dot{n}_{1}=2 \operatorname{Im} \sum_{234}\left(V_{11} a_{1}^{*} a_{2}^{*} a_{3} a_{4}+V_{12} a_{1}^{*} b_{2}^{*} b_{3} a_{4}\right) \delta_{1 ; 3+4-2}, \\
& \dot{l}_{1}=2 \operatorname{Im} \sum_{234}\left(V_{12} b_{1}^{*} a_{2}^{*} a_{3} b_{4}+V_{22} b_{1}^{*} b_{2}^{*} b_{3} b_{4}\right) \delta_{1 ; 3+4-2} .
\end{aligned}
$$

We shall exclude the terms with coinciding momenta and, in view of the initial conditions (3), take an ensemble average of Eqs. (6-7). To obtain the non-vanishing contribution from the correlation functions $Q_{1234}=$ $\left\langle a_{1}^{*} a_{2}^{*} a_{3} a_{4}\right\rangle, L_{1234}=\left\langle a_{1}^{*} b_{2}^{*} b_{3} a_{4}\right\rangle, M_{1234}=\left\langle b_{1}^{*} a_{2}^{*} a_{3} b_{4}\right\rangle$, and $P_{1234}=\left\langle b_{1}^{*} b_{2}^{*} b_{3} b_{4}\right\rangle$ we differentiate them with respect to time and use Eqs. (4-5). The resulting equations contain six-order correlation functions that simplify to the expressions in terms of the occupation numbers after the application of Wick's theorem and using $\mathbf{k}_{1}+\mathbf{k}_{2}=\mathbf{k}_{3}+\mathbf{k}_{4}$, since only these terms contribute to Eqs. (6-7). The differential equations for $Q_{1234}, L_{1234}, M_{1234}$ and $P_{1234}$ take the form

$$
\begin{aligned}
\dot{Q}_{1234} & =i \Delta_{11} \epsilon Q_{1234}+i 2 \tilde{Q}_{1234}, \\
\dot{P}_{1234} & =i \Delta_{22} \epsilon P_{1234}+i 2 \tilde{P}_{1234}, \\
\dot{L}_{1234} & =i \Delta_{12} \epsilon L_{1234}+i \tilde{L}_{1234}, \\
\dot{M}_{1234} & =i \Delta_{21} \epsilon M_{1234}+i \tilde{M}_{1234},
\end{aligned}
$$

where

$$
\begin{aligned}
\tilde{Q}_{1234} & =V_{11}\left[n_{2} n_{3} n_{4}+n_{1} n_{3} n_{4}-n_{1} n_{2} n_{3}-n_{1} n_{2} n_{4}\right], \\
\tilde{P}_{1234} & =V_{22}\left[l_{2} l_{3} l_{4}+l_{1} l_{3} l_{4}-l_{1} l_{2} l_{3}-l_{1} l_{2} l_{4}\right], \\
\tilde{L}_{1234} & =V_{12}\left[l_{2} l_{3} n_{4}+n_{1} l_{3} n_{4}-n_{1} l_{2} l_{3}-n_{1} l_{2} n_{4}\right] \\
\tilde{M}_{1234} & =V_{12}\left[n_{2} n_{3} l_{4}+l_{1} n_{3} l_{4}-l_{1} n_{2} n_{3}-l_{1} n_{2} l_{4}\right]
\end{aligned}
$$

and $\Delta_{i j} \epsilon=\left(k_{1}^{2} / m_{i}+k_{2}^{2} / m_{j}-k_{3}^{2} / m_{j}-k_{4}^{2} / m_{i}\right) / 2$. The kinetic approach is valid if the frequencies of the oscillations of the phases of the complex 


\section{Two-Component Bose Condensates}

amplitudes $a_{\mathbf{k}}$ and $b_{\mathbf{k}}$ are much larger than the energies of the non-linear interactions, therefore, we can assume that the energies $\epsilon_{i}$ are much larger than the inverse time of the variations of the functions $\tilde{Q}, \tilde{P}, \tilde{L}$, and $\tilde{M}$. This implies that the solutions of Eqs. (8) are

$$
\begin{aligned}
Q_{1234} & \approx 2 \pi \tilde{Q}_{1234} \delta\left(\Delta_{11} \epsilon\right), \\
P_{1234} & \approx 2 \pi \tilde{P}_{1234} \delta\left(\Delta_{22} \epsilon\right), \\
L_{1234} & \approx \pi \tilde{L}_{1234} \delta\left(\Delta_{12} \epsilon\right), \\
M_{1234} & \approx \pi \tilde{M}_{1234} \delta\left(\Delta_{21} \epsilon\right) .
\end{aligned}
$$

Finally, we replace the summation over momenta with integration to arrive at the kinetic equations for the smooth ensemble averaged values of the occupation numbers

$$
\begin{aligned}
\dot{n}_{1}= & \int \frac{d \mathbf{k}_{2} d \mathbf{k}_{3}}{32 \pi^{5}}\left(2 \delta\left(\Delta_{11} \epsilon\right) V_{11}^{2}\left[\left(n_{1}+n_{2}\right) n_{3} n_{4}-n_{1} n_{2}\left(n_{3}+n_{4}\right)\right]\right. \\
& \left.+\delta\left(\Delta_{12} \epsilon\right) V_{12}^{2}\left[\left(n_{1}+l_{2}\right) l_{3} n_{4}-n_{1} l_{2}\left(l_{3}+n_{4}\right)\right]\right), \\
\dot{l}_{1}= & \int \frac{d \mathbf{k}_{2} d \mathbf{k}_{3}}{32 \pi^{5}}\left(2 \delta\left(\Delta_{22} \epsilon\right) V_{22}^{2}\left[\left(l_{1}+l_{2}\right) l_{3} l_{4}-l_{1} l_{2}\left(l_{3}+l_{4}\right)\right]\right. \\
& \left.+\delta\left(\Delta_{21} \epsilon\right) V_{12}^{2}\left[\left(l_{1}+n_{2}\right) n_{3} l_{4}-l_{1} n_{2}\left(n_{3}+l_{4}\right)\right]\right),
\end{aligned}
$$

where $\mathbf{k}_{4} \equiv \mathbf{k}_{1}+\mathbf{k}_{2}-\mathbf{k}_{3}$.

The kinetic equations (11-12) describe the evolution of the wave spectrum towards the Rayleigh-Jeans equilibrium distribution ${ }^{13}$

$$
n_{k}^{\mathrm{eq}}=\frac{T}{k^{2}-\mu_{1}} \quad l_{k}^{\mathrm{eq}}=\frac{T}{k^{2}-\mu_{2}},
$$

where $\mu_{i}$ is the chemical potential of $i$-th gas. The parameter $T$ can be associated with the temperature at equilibrium. It depends on the initial states of the gases and can be found by fitting the distribution of the occupation numbers obtained from numerical integration of Eqs.(11)-(12) to Eq.(13). Dissipation, diffusion or viscosity introduce a frequency ultraviolet cut-off of large wavelength and therefore regularize these formally divergent at $k \rightarrow \infty$ solutions. The numerical simulations introduce a frequency cut-off through the spatial discretization.

Similar to the case of the condensation of one-component Bose gas ${ }^{13}$, 8 we are interested in the evolution of the singular particle distribution

$$
\begin{aligned}
n_{\mathbf{k}} & =n_{0}(t) \delta(\mathbf{k})+\phi_{1 \mathbf{k}}(t), \\
l_{\mathbf{k}} & =l_{0}(t) \delta(\mathbf{k})+\phi_{2 \mathbf{k}}(t),
\end{aligned}
$$




\section{Natalia G. Berloff and Chen Yin}

where $n_{0}$ and $l_{0}$ are the number of particles in condensates and $\phi_{i \mathbf{k}}$ are the uncondensed particles. In the equilibrium we expect

$$
\phi_{i \mathbf{k}} \approx \frac{T}{k^{2}},
$$

which corresponds to the distribution (13) with zero chemical potentials.

To see how the nonlinear interactions change this distribution we performed large scale numerical simulations of the dimensionless form of Eqs. (1) starting with a strongly nonequilibrium initial condition (3). The numerical scheme involves the fourth-order finite differences discretization in space and the fourth order Runge-Kutta in time, so is globally 4th order accurate. This scheme corresponds to the Hamiltonian system in the discrete variables $\psi_{i}^{j k n}$, where $i=1,2$ such that

$$
i \dot{\psi}_{i}^{j k n}=\partial H / \partial \psi_{i}^{j k n *}
$$

where

$$
\begin{aligned}
H= & \sum_{j k n} \sum_{i}\left(\psi _ { i } ^ { j k n * } \left[\frac { 1 } { 1 2 } \left(\psi_{i}^{j+2, k, n}+\psi_{i}^{j-2, k, n}+\psi_{i}^{j, k+2, n}\right.\right.\right. \\
& \left.+\psi_{i}^{j, k-2, n}+\psi_{i}^{j, k, n+2}+\psi_{i}^{j, k, n-2}\right)-\frac{4}{3}\left(\psi_{i}^{j+1, k, n}\right. \\
& \left.\psi_{i}^{j-1, k, n}+\psi_{i}^{j, k+1, n}+\psi_{i}^{j, k-1, n}+\psi_{i}^{j, k, n+1}+\psi_{i}^{j, k, n-1}\right) \\
& \left.\left.+\frac{15}{2} \psi_{i}^{j k n}\right]+\frac{1}{2} V_{i i}\left|\psi_{i}^{j k n}\right|^{4}\right)+V_{12} \prod_{i}\left|\psi_{i}^{j k n}\right|^{2},
\end{aligned}
$$

where it is understood that $V_{i i^{\prime}}$ are now the dimensionless coupling constants, $d x=d y=d z=1$ and $j, k, n=1, \ldots, N$. In the numerical simulations we use $d t=0.01$ and $N=64$ or $N=128$. The kinetic equations (11-12) conserve the number of particles $N_{1}=V \int n_{\mathbf{k}}(t) d \mathbf{k}, N_{2}=V \int l_{\mathbf{k}}(t) d \mathbf{k}$ and the kinetic energies of each gas $E_{1}=V \int k^{2} n_{\mathbf{k}}(t) d \mathbf{k}$ and $E_{2}=V \int k^{2} l_{\mathbf{k}}(t) d \mathbf{k}$. In the numerical simulations we had to decrease the number of parameters in the system by considering equal masses and equal intercomponent interaction constants. The final number of particles in the condensates depends strongly on the energy $H$ of the system and on the total number of particles in each condensate. Our numerical simulations confirmed the existence of the condensation process for sufficiently low energy densities. On FIG.1 we show the time evolution of the number of particles in condensates for $V_{12} / V_{i i}=0.1$, $H / V=1, N_{1} / V=1 / 2$, and $N_{2} / V=1 / 4$ in comparison with the case of the condensation of two gasses starting with the same initial condition, such that $E_{\text {kin1 }}=2 E_{\text {kin2}}$, but with no interaction between particles of different gasses, so that $V_{12}=0$. The number of particles in the first condensate is increased and the number of particles in the second condensate is decreased due to 


\section{Two-Component Bose Condensates}

Fig. 1. (colour online) The time evolution of the density of the condensed particles $n_{0} / N_{1}$ (left panel) and $l_{0} / N_{2}$ (right panel) for $H / V=2, N_{1} / V=$ $1 / 2, N_{2} / V=1 / 4$. Black lines give the density of the condense particles in the presence of the intracoupling interactions $V_{12} / V_{i i}=0.1$. Red lines correspond to the case of two gasses with no intracoupling interactions $V_{12}=$ 0 , where the initial kinetic energies of two gases satisfy $E_{\mathrm{kin} 1}=2 E_{\mathrm{kin} 2}$.
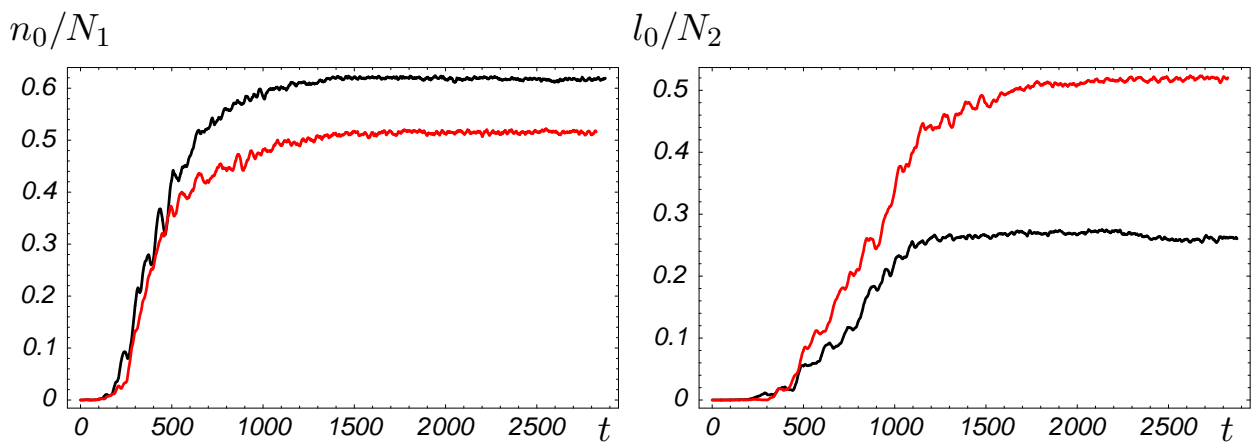

interactions. We found this effect to be more pronounced for intermediate energies and when $N_{2} / N_{1} \approx 1 / 2$.

The condensate fractions as functions of the total energy density $H / V$ are plotted on FIG.2. Note that the presence of interactions between gases increases (decreases) the largest value of energy for which the condensation occurs in the gas with the larger (smaller) number of particles. The reason for such striking change in condensation is that if two gasses do not interact $\left(V_{12}=0\right)$, each gas reaches the equilibrium characterized by its own temperature that depends on the total density and energy of that gas alone. In the presence of interactions, however, both gasses equilibrate at the same temperature (15) which lies between temperatures of two isolated gases in equilibrium. As the result, the number of condensed particles increases for one gas and decreases for the other gas.

When both gases condense, the system passes through the stages of weak turbulence to superfluid turbulence with a tangle of topological defects (see FIG. 3) previously identified in the mixtures of two BECs ${ }^{14}$ as vortex ring-vortex ring and slaved wave - vortex ring complexes clearly seen on the last time snapshot of FIG.3. The number of various complexes generated during condensation greatly varies depending on the initial state of the system. Complexes interact among each other and change from one type to another. For instance, a vortex ring - vortex ring complex may lose energy to sound waves and move to a lower branch in $p \mathcal{E}$-space shown in FIG.4 to become a vortex ring - slaved wave complex. Alternatively, two vortex ring 


\section{Natalia G. Berloff and Chen Yin}

Fig. 2. (colour online) Condensate fractions $n_{0} / N_{1}$ (black solid line) and $l_{0} / N_{2}$ (green solid line) as functions of the total energy density $H / V$ for $N_{1} / V=1 / 2, N_{2} / V=1 / 4$, and $V_{12} / V_{i i}=0.1$. Condensate fractions for two gases with $V_{12} / V_{i i}=0$ are shown by dashed lines. The initial kinetic energies of two gases satisfy $E_{\mathrm{kin} 1}=2 E_{\mathrm{kin} 2}$.

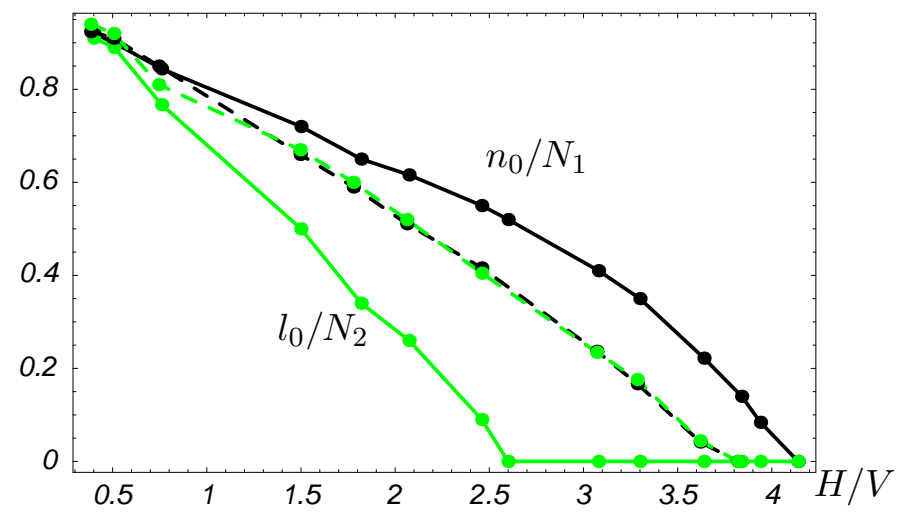

- slaved wave complexes may interact transferring energy from one complex to another, so that one of the complexes gains energy and becomes a vortex ring - vortex ring. A similar exchange of energy occurs between two rarefaction pulses of one component condensate leading to a creation of a vortex ring 15 .

The evolution of the system is reminiscent of the Kibble-Zurek scenario ${ }^{16}$ of a formation of the topological defects when the system is quickly quenched below the point of the second-order transition. The process that we have just described would correspond to the formation of the cosmological vortons and springs that are analogous to the vortex ring-slaved wave and vortex ring-vortex ring complexes correspondingly ${ }^{14}$. The similarity between these types of defects is topological: consider a two component system which is described by two complex scalar fields $\psi_{1}$ and $\psi_{2}$ with an approximate $U(2)$ symmetry. This symmetry can be broken down to form string loops (vortices) in $\psi_{1}$ with $\psi_{2}$ condensing inside the core. This construction would correspond to a vorton. If $\psi_{2}$ field condenses as a string loop inside the core of a vortex formed by $\psi_{1}$ it becomes analogous to a spring defect. From these topological consideration the complexes we consider here are non-relativistic counterparts of topological defects extensively studied in high-density QCD, cosmology and high-temperature superconductivity. 


\section{Two-Component Bose Condensates}

Fig. 3. (colour online) Evolution of topological defects in the long-wavelength parts $\tilde{\psi}_{i}$, with $i=1$ (green, light grey) and $i=2$ (red, dark grey) of the fields $\psi_{i}$ in the computational box $128^{3}$. The defects are visualised by isosurfaces $\left|\tilde{\psi}_{i}\right|^{2}=0.04\left\langle\left|\tilde{\psi}_{i}\right|^{2}\right\rangle$. High-frequency spatial waves are suppressed by the factor $\max \left\{1-k^{2} / k_{c}^{2}, 0\right\}$, where the cut-off wave number is chosen according to the phenomenological formula $k_{c}=9-t / 1000$.
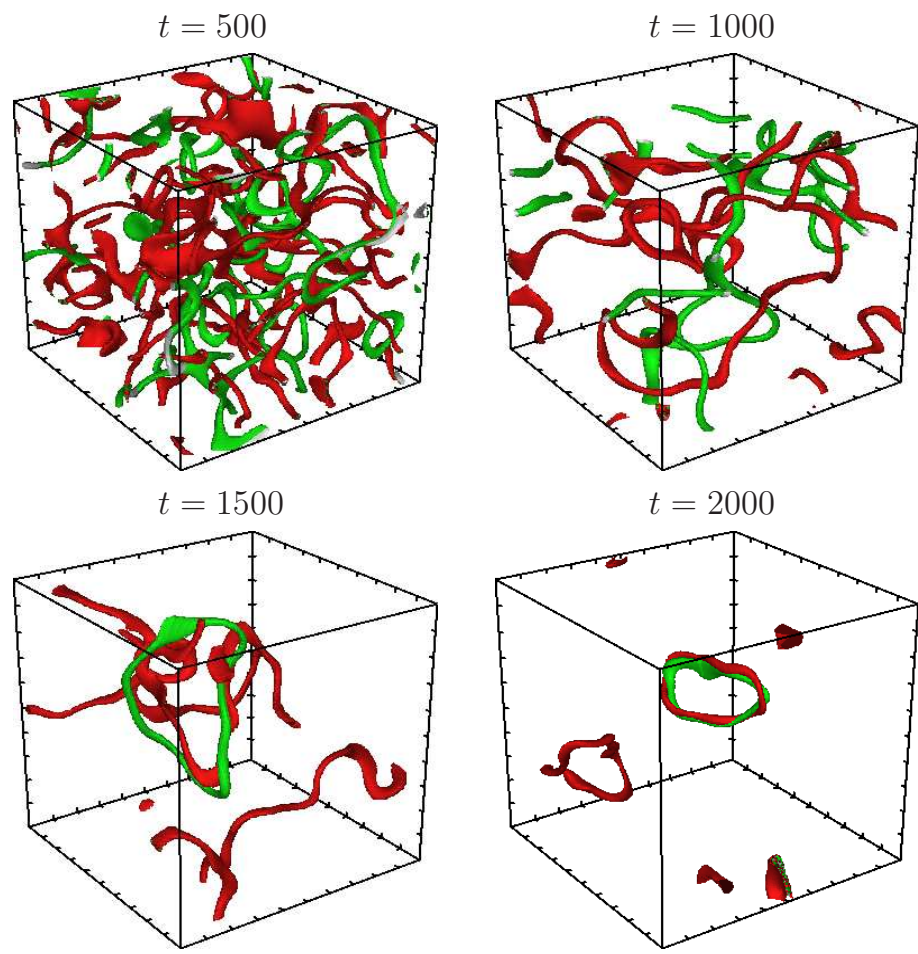

\section{COHERENT STATES}

In this section we study the solitary wave solutions of the coupled GP model (1). Many solitary wave structures have been recently identified in two-component one-dimensional BECs ${ }^{18}$ such as bound dark-dark, darkbright, dark-antidark, dark-grey, etc. complexes. In higher dimensions, domain walls ${ }^{19}$ and skyrmions (vortons) ${ }^{20}$ have been found. Previously we 14 determined the complete families of solitary waves in miscible condensates in the case of equal masses and intracomponent scattering lengths. In what follows we extent these results to a more general case of nonequal masses, nonequal coupling constants and to $2 \mathrm{D}$.

To study the equilibrium properties the energy functional (2) has to be 


\section{Natalia G. Berloff and Chen Yin}

minimized subject to the constraints on the conservation of particles leading to introduction of two chemical potentials $\mu_{1}=V_{11} n_{1}+V_{12} n_{2}, \mu_{2}=$ $V_{12} n_{1}+V_{22} n_{2}$, where $n_{i}=\left|\psi_{i}\right|^{2}$ is the number density in equilibrium. The dispersion relation between the frequency $\omega$ and the wave number $k$ of the linear perturbations $(\propto \exp [i \mathbf{k} \cdot \mathbf{x}-i \omega t])$ around homogeneous states is obtained as

$$
\left(\omega^{2}-\omega_{1}^{2}\right)\left(\omega^{2}-\omega_{2}^{2}\right)=\omega_{12}^{4}
$$

where $\omega_{i}^{2}(k)=c_{i}^{2} k^{2}+\hbar^{2} k^{4} / 4 m_{i}^{2}$ coincides with a one-component Bogoliubov spectrum with the customary defined sound velocity $c_{i}^{2}=n_{i} V_{i i} / m_{i}$ and $\omega_{12}^{2}=c_{12}^{2} k^{2}$ where $c_{12}^{2}=n_{1} n_{2} V_{12}^{2} / m_{1} m_{2}$. The system is dynamically stable if the spectrum (18) is real and positive which implies that $V_{11} V_{22}>V_{12}^{2}$, $V_{i i}>0$ for stability ${ }^{12}$. The acoustic branches of Eq. (18) are $\omega_{ \pm} \approx c_{ \pm} k$ with the corresponding sound velocities

$$
2 c_{ \pm}^{2}=c_{1}^{2}+c_{2}^{2} \pm \sqrt{\left(c_{1}^{2}-c_{2}^{2}\right)^{2}+4 c_{12}^{4}} .
$$

The solitary waves we seek below are all subsonic, so their velocity $U$ is less than $c_{-}$.

A dimensionless form of (1) is obtained by introducing the chemical potentials $\psi_{i} \rightarrow \psi_{i} \exp \left[-\mathrm{i} \mu_{i} t / \hbar\right]$ and using dimensionless units

$$
\mathbf{x} \rightarrow \frac{\hbar}{\left(2 m_{1} \mu_{1}\right)^{1 / 2}} \mathbf{x}, \quad t \rightarrow \frac{\hbar}{2 \mu_{1}} t, \quad \psi_{i} \rightarrow \sqrt{\frac{\mu_{1}}{V_{11} n_{i}}} \psi_{i} .
$$

This leads to the system of nonlinear Schrödinger equations

$$
\begin{aligned}
-2 i \frac{\partial \psi_{1}}{\partial t}= & \nabla^{2} \psi_{1}+\left(1-\left|\psi_{1}\right|^{2}-\alpha_{1}\left|\psi_{2}\right|^{2}\right) \psi_{1} \\
-2 i \frac{\partial \psi_{2}}{\partial t}= & \gamma \nabla^{2} \psi_{2}+\left(1-\alpha_{1}\left|\psi_{1}\right|^{2}-\frac{\alpha_{1}}{\alpha_{2}}\left|\psi_{2}\right|^{2}-\Lambda^{2}\right) \psi_{2}, \\
& \psi_{1} \rightarrow \psi_{1 \infty}, \quad \psi_{2} \rightarrow \psi_{2 \infty}, \quad \text { as } \quad|\mathbf{x}| \rightarrow \infty
\end{aligned}
$$

where $\alpha_{i}=V_{12} / V_{i i}, \gamma=m_{1} / m_{2}$ and $\Lambda^{2}=\left(\mu_{1}-\mu_{2}\right) / \mu_{1}$ is the measure of asymmetry between chemical potentials (where we assume that $\mu_{1}>\mu_{2}$ ). Note that the use of Feshbach resonances to vary the interactions between atoms makes this entire range of parameters experimentally accessible. The condition of dynamic stability becomes $\alpha_{1} \alpha_{2}<1$. To also ensure stability against collapse when only the density of one component is varied, the final stability criterion becomes

$$
0<\alpha_{1} \alpha_{2}<1
$$

The acoustic branches (19) are

$$
c_{ \pm}^{2}=\frac{1}{2}\left(\psi_{1 \infty}^{2}+\frac{\alpha_{1}}{\alpha_{2}} \gamma \psi_{2 \infty}^{2} \pm \sqrt{\left(\psi_{1 \infty}^{2}-\frac{\alpha_{1}}{\alpha_{2}} \gamma \psi_{2 \infty}^{2}\right)^{2}+4 \alpha_{1}^{2} \gamma \psi_{1 \infty}^{2} \psi_{2 \infty}^{2}}\right) .
$$




\section{Two-Component Bose Condensates}

To find solitary wave solutions moving with velocity $U$ in positive $z$-direction, we solved

$$
\begin{aligned}
2 \mathrm{i} U \frac{\partial \psi_{1}}{\partial z}= & \nabla^{2} \psi_{1}+\left(1-\left|\psi_{1}\right|^{2}-\alpha_{1}\left|\psi_{2}\right|^{2}\right) \psi_{1} \\
2 \mathrm{i} U \frac{\partial \psi_{2}}{\partial z}= & \gamma \nabla^{2} \psi_{2}+\left(1-\alpha_{1}\left|\psi_{1}\right|^{2}-\frac{\alpha_{1}}{\alpha_{2}}\left|\psi_{2}\right|^{2}-\Lambda^{2}\right) \psi_{2} \\
& \psi_{1} \rightarrow \psi_{1 \infty}, \quad \psi_{2} \rightarrow \psi_{2 \infty}, \quad \text { as } \quad|\mathbf{x}| \rightarrow \infty
\end{aligned}
$$

We are interested in the case when both condensates have nonzero uniform states. The values of the wave-functions of the solitary waves at infinity in (21) are given by

$$
\begin{aligned}
& \psi_{1 \infty}^{2}=\left(1-\alpha_{2}+\alpha_{2} \Lambda^{2}\right) /\left(1-\alpha_{1} \alpha_{2}\right) \\
& \psi_{2 \infty}^{2}=\frac{\alpha_{2}}{\alpha_{1}}\left(1-\alpha_{1}-\Lambda^{2}\right) /\left(1-\alpha_{1} \alpha_{2}\right)
\end{aligned}
$$

so that we will assume that

$$
\alpha_{1}<1-\Lambda^{2}, \quad \alpha_{2}<\frac{1}{1-\Lambda^{2}} .
$$

In a compact form the equations (24) can be written as

$$
2 \mathrm{i} U \frac{\partial \psi_{i}}{\partial z}=\frac{m_{1}}{m_{i}} \nabla^{2} \psi_{i}+f_{i}\left(\psi_{1}, \psi_{2}\right) \psi_{i}, \quad i=1,2
$$

where we used the notation

$$
\begin{aligned}
& f_{1}\left(\psi_{1}, \psi_{2}\right)=1-\left|\psi_{1}\right|^{2}-\alpha_{1}\left|\psi_{2}\right|^{2} \\
& f_{2}\left(\psi_{1}, \psi_{2}\right)=1-\alpha_{1}\left|\psi_{1}\right|^{2}-\frac{\alpha_{1}}{\alpha_{2}}\left|\psi_{2}\right|^{2}-\Lambda^{2}
\end{aligned}
$$

\subsection{Integral Identities}

Each solitary wave complex that belongs to a family of the solitary wave solutions for a chosen set of $\left(\alpha_{1}, \alpha_{2}, \gamma, \Lambda^{2}\right)$ is characterised by its velocity, $U$, vortex radii $b_{i}$, momenta $\mathbf{p}_{i}=\left(0,0, p_{i}\right)$, and energy. The impulse of the $i$-th component ${ }^{14}$ is

$$
\mathbf{p}_{i}=\frac{1}{2 i} \int\left[\left(\psi_{i}^{*}-\psi_{i \infty}\right) \nabla \psi_{i}-\left(\psi_{i}-\psi_{i \infty}\right) \nabla \psi_{i}^{*}\right] d V .
$$

We form the energy, $\mathcal{E}$, by subtracting the energy of an undisturbed system of the same mass for which $\psi_{i}=$ const everywhere, from the energy of the 


\section{Natalia G. Berloff and Chen Yin}

system with a solitary wave, so that the energy of the system becomes

$$
\begin{aligned}
\mathcal{E} & =\frac{1}{2} \int\left\{\left|\nabla \psi_{1}\right|^{2}+\gamma\left|\nabla \psi_{2}\right|^{2}+\frac{1}{2}\left(\psi_{1 \infty}^{2}-\left|\psi_{1}\right|^{2}\right)^{2}\right. \\
& \left.+\frac{\alpha_{1}}{2 \alpha_{2}}\left(\psi_{2 \infty}^{2}-\left|\psi_{2}\right|^{2}\right)^{2}\right\} d V+\frac{\alpha_{1}}{2} \int \prod_{i=1}^{2}\left(\psi_{i \infty}^{2}-\left|\psi_{i}\right|^{2}\right) d V
\end{aligned}
$$

Alternatively, we can write

$$
\mathcal{E}=\frac{1}{2} \int \sum\left\{\frac{m_{1}}{m_{i}}\left|\nabla \psi_{i}\right|^{2}+\frac{1}{2} f_{i}\left(\psi_{1}, \psi_{2}\right)\left(\psi_{i \infty}^{2}-\left|\psi_{i}\right|^{2}\right)\right\} d V
$$

By performing the variation $\psi_{i} \rightarrow \psi_{i}+\delta \psi_{i}$ in the integrals for $\mathcal{E}$ and $p_{i}$ and discarding surface integrals that vanish provided $\delta \psi_{i} \rightarrow 0$ for $|\mathbf{x}| \rightarrow \infty$ one gets

$$
\begin{aligned}
\delta p_{i} & =\frac{1}{\mathrm{i}} \int\left(\delta \psi_{i}^{*} \frac{\partial \psi_{i}}{\partial z}-\delta \psi \frac{\partial \psi_{i}^{*}}{\partial z}\right) d V \\
\delta \mathcal{E} & =-\frac{1}{2} \int \sum_{i=1}^{2} \delta \psi_{i}^{*}\left(\frac{m_{1}}{m_{i}} \nabla^{2} \psi_{i}+f_{i}\left(\psi_{1}, \psi_{2}\right) \psi_{i}\right) \\
& +\delta \psi_{i}\left(\frac{m_{1}}{m_{i}} \nabla^{2} \psi_{i}^{*}+f_{i}\left(\psi_{1}, \psi_{2}\right) \psi_{i}^{*}\right) d V
\end{aligned}
$$

where the notation (29) is used. By making use of (24) and its complex conjugate, we obtain $\delta \mathcal{E}=U \delta\left(p_{1}+p_{2}\right)$, or $U=\partial \mathcal{E} / \partial\left(p_{1}+p_{2}\right)$, where the derivative is taken along the solitary wave sequence. The same expression is obeyed by the sequences of classical vortex rings in an incompressible fluid and by the solitary waves of one component GP equation ${ }^{21}$.

Next we replace $\psi_{i} \rightarrow \psi_{i}-\psi_{i \infty}$ in the first two terms of (31) and integrate by parts using (24) and (30) to simplify the resulting expressions. The result is the integral identity

$$
\mathcal{E}=U\left(p_{1}+p_{2}\right)+\frac{1}{4} \int \sum f_{i}\left(\psi_{1}, \psi_{2}\right)\left|\psi_{i \infty}-\psi_{i}\right|^{2} d V
$$

We multiply (24) by $s \partial \psi_{i}^{*} / \partial s$, the complex conjugate of (24) by $s \partial \psi_{i} / \partial s$ where $s=\sqrt{x^{2}+y^{2}}$ in $3 \mathrm{D}$ and $s=x$ in $2 \mathrm{D}$, and add. After some integration by parts we get

$$
\begin{aligned}
2 U\left(p_{1}+p_{2}\right) & =\int\left|\frac{\partial \psi_{1}}{\partial z}\right|^{2}+\gamma\left|\frac{\partial \psi_{2}}{\partial z}\right|^{2} d V-\int\left|\frac{\partial \psi_{1}}{\partial x}\right|^{2}+\gamma\left|\frac{\partial \psi_{2}}{\partial x}\right|^{2} d V \\
& +\frac{1}{2} \sum \int f_{i}\left(\psi_{1}, \psi_{2}\right)\left(\psi_{i \infty}^{2}-\left|\psi_{i}\right|^{2}\right) d V
\end{aligned}
$$




\section{Two-Component Bose Condensates}

in $2 \mathrm{D}$ and

$$
\begin{aligned}
2 U\left(p_{1}+p_{2}\right) & =\int\left|\frac{\partial \psi_{1}}{\partial z}\right|^{2}+\gamma\left|\frac{\partial \psi_{2}}{\partial z}\right|^{2} d V \\
& +\frac{1}{2} \sum \int f_{i}\left(\psi_{1}, \psi_{2}\right)\left(\psi_{i \infty}^{2}-\left|\psi_{i}\right|^{2}\right) d V
\end{aligned}
$$

in 3D. Similarly, we multiply (24) by $z \partial \psi_{i}^{*} / \partial z$, their complex conjugate by $z \partial \psi_{i}^{*} / \partial z$, add the resulting equations together and after integration by parts we get

$$
\begin{aligned}
\int\left|\frac{\partial \psi_{1}}{\partial z}\right|^{2}+\gamma\left|\frac{\partial \psi_{2}}{\partial z}\right|^{2} d V & =\int\left|\nabla_{\perp} \psi_{1}\right|^{2}+\gamma\left|\nabla_{\perp} \psi_{2}\right|^{2} d V \\
& +\frac{1}{2} \sum \int f_{i}\left(\psi_{1}, \psi_{2}\right)\left(\psi_{i \infty}^{2}-\left|\psi_{i}\right|^{2}\right) d V
\end{aligned}
$$

where $\nabla_{\perp}$ is the gradient orthogonal to $z$-axis. From (32) and (36) in $2 \mathrm{D}$ and (32) and (37) in 3D we get

$$
\mathcal{E}=\int\left|\frac{\partial \psi_{1}}{\partial z}\right|^{2}+\gamma\left|\frac{\partial \psi_{2}}{\partial z}\right|^{2} d V
$$

From (35), (36) and (39) we get

$$
\begin{aligned}
& U \sum p_{i}=\frac{1}{2} \int \sum f_{i}\left(\psi_{1}, \psi_{2}\right)\left(\psi_{i \infty}^{2}-\left|\psi_{i}\right|^{2}\right) d V \\
& \mathcal{E}=\frac{1}{4} \int \sum f_{i}\left(3 \psi_{i \infty}^{2}-\psi_{i \infty}\left(\psi_{i}+\psi_{i}^{*}\right)-\left|\psi_{i}\right|^{2}\right) d V
\end{aligned}
$$

in $2 \mathrm{D}$ and from (35), (37) and (39) we get

$$
\begin{aligned}
& \mathcal{E}=\int \sum_{i=1}^{2} f_{i}\left(\psi_{1}, \psi_{2}\right)\left(2 \psi_{i \infty}^{2}-\psi_{i \infty}\left(\psi_{i}+\psi_{i}^{*}\right)\right) d V \\
& 2 U \sum_{i=1}^{2} p_{i}=\int \sum_{i=1}^{2} f_{i}\left(\psi_{1}, \psi_{2}\right)\left(3 \psi_{i \infty}^{2}-\psi_{i \infty}\left(\psi_{i}+\psi_{i}^{*}\right)-\left|\psi_{i}\right|^{2}\right) d V
\end{aligned}
$$

in $3 \mathrm{D}$.

In the integrals above we understand that $d V=s d s d z$ in $3 \mathrm{D}$ and $d V=$ $d x d z$ in $2 \mathrm{D}$. These integral identities are used to check the numerical accuracy of the solutions. 


\section{Natalia G. Berloff and Chen Yin}

\subsection{Solitary Waves in $3 \mathrm{D}$}

Previously we ${ }^{14}$ obtained the families of solitary wave complexes in three dimensions for miscible two-component condensates. The axisymmetric solitary waves were found by rewriting (24) in cylindrical coordinates $(s, \theta, z)$ for the deviations from the solutions at infinity $\Psi_{i}=\psi_{i}-\psi_{i \infty}$. Stretched variables $z^{\prime}=z$ and $s^{\prime}=s \sqrt{1-2 U^{2}}$ were introduced and the infinite domain was mapped onto the box $\left(0, \frac{\pi}{2}\right) \times\left(-\frac{\pi}{2}, \frac{\pi}{2}\right)$ using the transformation $\widehat{z}=\tan ^{-1}\left(D z^{\prime}\right)$ and $\widehat{s}=\tan ^{-1}\left(D s^{\prime}\right)$, where $D \sim 0.4-0.5$. Transformed equations (24) were expressed in second-order finite difference form using $100^{2}$ grid points, and the resulting nonlinear equations were solved by Newton-Raphson iteration procedure using banded matrix linear solver based on bi-conjugate gradient stabilised iterative method with preconditioning. The solutions were classified according to the structure of the wavefunction in each component. Several families of solitary wave complexes were found: (1) vortex rings of various radii in each of the components (VR-VR complexes), (2) a vortex ring in one component coupled to a rarefaction solitary wave of the other component (VR-RP and RP-VR complexes), (3) two coupled rarefaction waves (RP-RP complexes), (4) either a vortex ring or a rarefaction pulse coupled to a localised disturbance of a very low momentum (slaved wave) (VR-SW, SW-VR, RP-SW, SW-RP complexes). The continuous families of such waves were shown in the momentum-energy plane for various values of the interaction strengths and the relative differences between the chemical potentials of two components. FIG.4 presents the dispersion curves of three families of the axisymmetric solitary wave solutions for $\gamma=1, \alpha_{1}=\alpha_{2}=0.1$ and $\Lambda^{2}=0.1$ and FIG.5 gives the density isosurface and density contour plots of a VR-VR solution with $\gamma=1, \alpha_{1}=\alpha_{2}=0.5$, $\Lambda^{2}=0.25$.

Next we consider non-equal masses $\gamma=2$, but keep intracomponent interaction potentials equal, $\alpha_{1}=\alpha_{2}$. This choice of parameters is close to experimental values for the mixture of ${ }^{41} \mathrm{~K}^{-87} \mathrm{Rb}{ }^{2}$. In experiments $m_{1} / m_{2}=$ $1.46^{2}$ and $a_{11} / a_{22}=99 / 60$ where $m_{1}, a_{11}$ and $m_{2}, a_{22}$ are masses and scattering lengths of $\mathrm{Rb}$ and $\mathrm{K}$ correspondingly. This gives $\gamma=2.13$ and $\alpha_{1} / \alpha_{2}=1.29$.

In comparison with the equal masses case, the solitary wave in the second component possesses significantly larger impulse (4-5 times larger for $U \sim 0.3-0.5)$ and the vortex ring of the second component has much larger radius (about 2-2.5 times larger for $U \sim 0.3-0.5$ ). FIG. 6 shows the isoplot of the VR-VR solution of (24) with $\alpha_{1}=\alpha_{2}=0.1, \Lambda^{2}=0.1$ and $\gamma=2$ that moves with $U=0.5$. In contrast with families depicted on FIG. 4, SW -VR family is now above $\operatorname{VR}(\mathrm{RP})-\mathrm{SW}$ family. The speed of sound is 


\section{Two-Component Bose Condensates}

Fig. 4. (colour online) The dispersion curves of three families of the axisymmetric solitary wave solutions of (24) with $\gamma=1, \alpha_{1}=\alpha_{2}=0.1$ and $\Lambda^{2}=0.1$. The numbers next to the dots give the velocity of the solitary wave solution. The top (black) branch corresponds to VR-VR (VR-RP for $U=0.58$ ) complexes. The middle (green) branch shows $p$ vs $\mathcal{E}$ for VR-SW complexes and the bottom (red) branch is the dispersion curve of SW-VR (SW-RP for $U=0.58$ ) complexes.

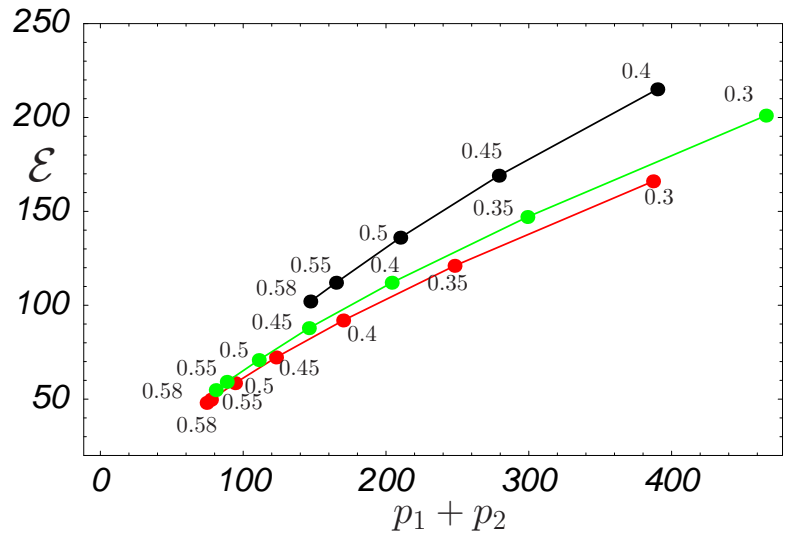

increased from 0.6169 for $\gamma=1$ to 0.6703 for $\gamma=2$. As a result there is a well-pronounced upper branch in each family. Remarkably, the vortex ring in the second component exists on the upper branch in both $\mathrm{VR}(\mathrm{RP})-\mathrm{VR}$ family and SW-VR family. There are no vortex rings on the upper branch in one-component condensates.

Finally, we considered the case of non-equal intracomponent interaction potentials of equal masses. The parameters we used were $\alpha_{1}=0.2, \alpha_{2}=$ $0.1, \Lambda^{2}=0.1$ and $\gamma=1$. This time the radii of the vortex rings in two components coincide to two significant digits with the radii of the vortex rings if $\alpha_{1}=0.1$, but the impulse of the second component is increased by a factor of about 2 .

\subsection{Solitary Waves in 2D}

For completeness we obtained the solitary waves of the coupled GP model (24) in two dimensions. In the limit $\alpha \rightarrow 0$, two components become uncoupled, in which case the solitary wave sequence for each component follows the dispersion curve of the one-component GP equation ${ }^{21}$. The family of solitary waves in $2 \mathrm{D}$ is represented by a pair of point vortices (VP) of 


\section{Natalia G. Berloff and Chen Yin}

Fig. 5. (colour online) Density isosurface (left panel) and density contour plots (middle and right panels) at $\left|\psi_{1}\right|^{2}=\frac{1}{10} \psi_{1 \infty}^{2}$ and $\left|\psi_{2}\right|^{2}=\frac{1}{10} \psi_{2 \infty}^{2}$ for the VR-VR complex for $\gamma=1, \alpha_{1}=\alpha_{2}=0.5, \Lambda^{2}=0.25$ that is moving with $U=0.3$. The radii are $b_{1}=5.194$ and $b_{2}=4.796$.
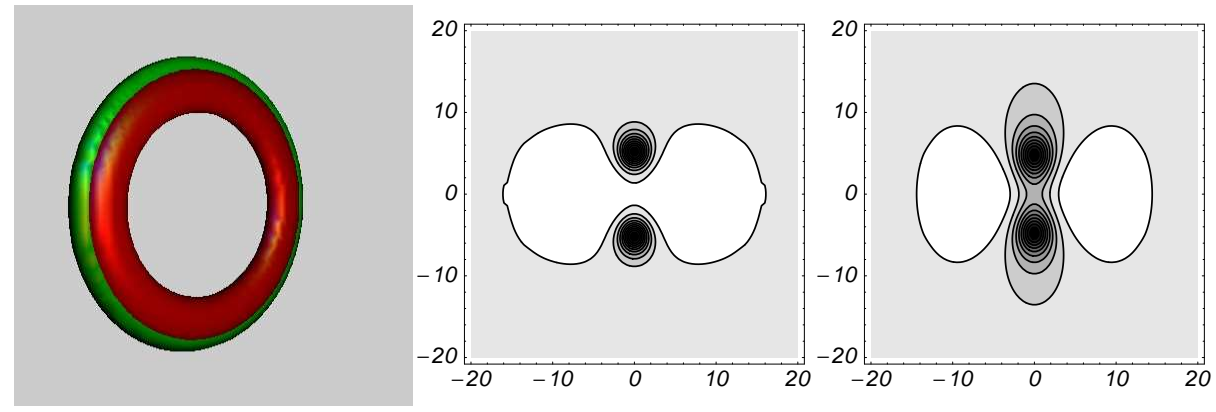

opposite circulation if $U_{c}<0.56 c_{i}$. These vortices are separated by distance $2 b_{i} \sim U^{-1}$ for small $U$. As the velocity increases, the wave loses its vorticity and becomes a rarefaction pulse. As $U \rightarrow c_{i}$ both energy and momentum per unit length approach zero and the solutions asymptotically approach the $2 \mathrm{D}$ rational solution of Kadomtsev-Petviashvili Type I equation. The sequence merges tangentially with the phonon branch of the dispersion curve in each of the uncoupled components. For $\alpha \neq 0$ we have $c_{1} \neq c_{2}$, so different components become RPs at different critical values of $U$ and a variety of complexes becomes possible. Table 1 gives an example of various transitions from one complex to another as the velocity $U$ increases in the system with $\alpha=0.05$ and $\Lambda^{2}=0.1$.

Table 1. The velocity, $U$, energy, $\mathcal{E}$, momenta, $p_{i}$, and half-separations between centres of the point vortices, $b_{i}$, of the solitary wave solutions of Eqs. (24) with $\alpha=0.05$ and $\Lambda^{2}=0.1$. The sequence terminates at $U=c_{-} \approx 0.646$.

\begin{tabular}{ccccccc}
\hline \hline$U$ & $\mathcal{E}$ & $p_{1}$ & $p_{2}$ & $b_{1}$ & $b_{2}$ & complex \\
\hline 0.40 & 14.7 & 13.8 & 12.1 & 0.915 & 0.498 & VP-VP \\
0.43 & 13.7 & 12.5 & 10.9 & 0.184 & - & VP-RP \\
0.45 & 13.0 & 11.7 & 10.2 & - & - & RP-RP \\
0.5 & 11.4 & 9.90 & 8.46 & - & - & RP-RP \\
0.6 & 7.68 & 6.29 & 5.36 & - & - & RP-RP \\
\hline \hline
\end{tabular}

Next we will consider the cases of more intermediate values of intercoupling interaction strength. Similarly to the three-dimensional case, in addition to the VP-VP, VP-RP, and RP-RP complexes, there are new classes of solitary waves that have no analog in one-component condensates. In these complexes the disturbance of a very low impulse (slaved wave, SW) in one 


\section{Two-Component Bose Condensates}

Fig. 6. (colour online) The dispersion curves of three families of the axisymmetric solitary wave solutions of (24) with $\gamma=2, \alpha_{1}=\alpha_{2}=0.1$ and $\Lambda^{2}=0.1$ (left panel). The numbers next to the dots give the velocity of the solitary wave solution. The top (black) branch corresponds to VR-VR or VR-RP complexes. The middle (red) branch is the dispersion curve of $\mathrm{SW}-\mathrm{VR}$ and SW-RP complexes. The bottom (green) branch shows $p$ vs $\mathcal{E}$ for VR-SW complexes. On the right panel the density isoplot of the VR-VR solution moving with $U=0.5$ is shown.
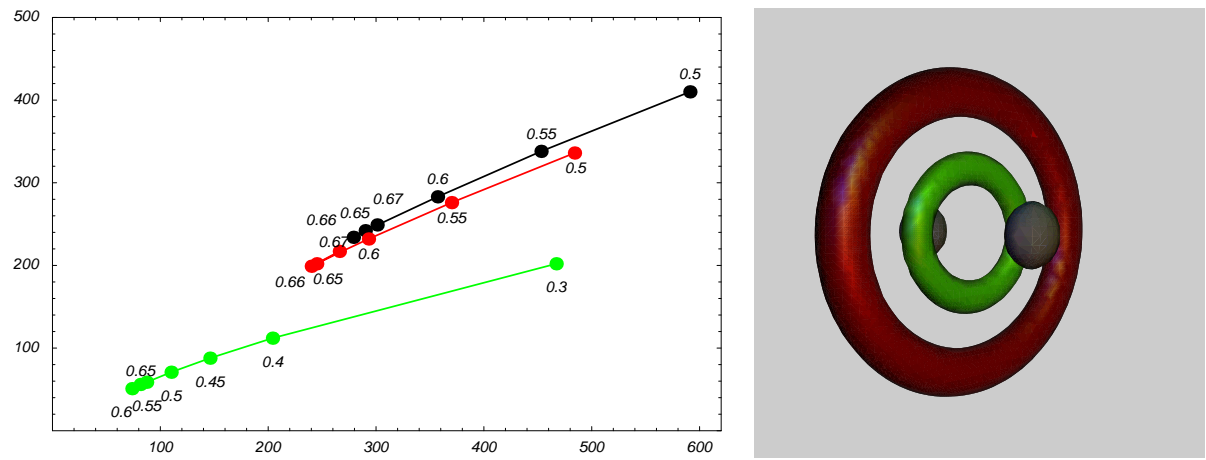

condensate is dragged by either VP or RP structure of the other component. The density of SW is maximal where the density of either VP or RP is minimal and vise versa. For fixed values of $\alpha_{1}, \alpha_{2}$ and $\Lambda^{2}$ the system has three families of solitary wave complexes: VP(RP)-VP(RP), SW-VP(RP) and $\mathrm{VP}(\mathrm{RP})-\mathrm{SW}$ as FIG. 7 illustrates. Also, FIG.7 shows the dispersion curves of several other families of the solitary wave solutions in the system when two out of three parameters $\left(\alpha_{1}=\alpha_{2}, \Lambda^{2}\right.$ and $\left.U\right)$ are kept fixed.

\section{SOLITARY WAVES ON A VORTEX LINE}

Similar to the one-component condensate ${ }^{23}$, two-component condensates can support families of solitary waves that travel along the topological defects. We shall use the ansatz

$$
\begin{aligned}
& \psi_{1}=\left(R_{1}(s)+\chi_{1}(s, z)\right) \exp (i \theta), \\
& \psi_{2}=R_{2}(s)+\chi_{2}(s, z),
\end{aligned}
$$

in the coupled GP model (24), where $R_{1}(s)$ is the amplitude of the straight line vortex of the first component of one unit of circulation placed along the $z$-axis, $R_{2}(s)$ is the amplitude of the second component and $\chi_{i}$ are the localized disturbances moving with a constant velocity $U$ in the positive 


\section{Natalia G. Berloff and Chen Yin}

Fig. 7. (colour online) The dispersion curves of several families of the solitary wave solutions of Eqs. (24). The solid lines show three families of solutions with $\alpha_{1}=\alpha_{2}=0.5$ and $\Lambda^{2}=0.1$. The numbers next to the dots give the velocity of the solitary wave. The top (black) branch corresponds to VP-VP complexes. The middle (green) branch shows $p$ vs $\mathcal{E}$ for VP-SW complexes and the bottom (red) branch is the dispersion curve of SW-VP complexes. The dashed (blue) line across the solid black branch shows the VP-VP complexes for $U=0.2, \Lambda^{2}=0.1$ as the intercoupling parameter $\alpha_{1}=\alpha_{2}$ increases in increments of 0.1 from 0.1 (top point) to 0.7 (bottom point). The light grey (magenta) dashed line shows the VP-VP complexes for $U=0.2$ and $\alpha_{1}=\alpha_{2}=0.5$ with the asymmetry parameter $\Lambda^{2}$ taking values 0.05 (top point), 0.1,0.2,0.3 (bottom point). The insets show the plots of $z=\left|\psi_{1}(x, y)\right|^{2}$ (top) and $z=\left|\psi_{2}(x, y)\right|^{2}$ (bottom) for the VP-SW complex with $U=0.2, \alpha_{1}=\alpha_{2}=0.5, \Lambda^{2}=0.1$.

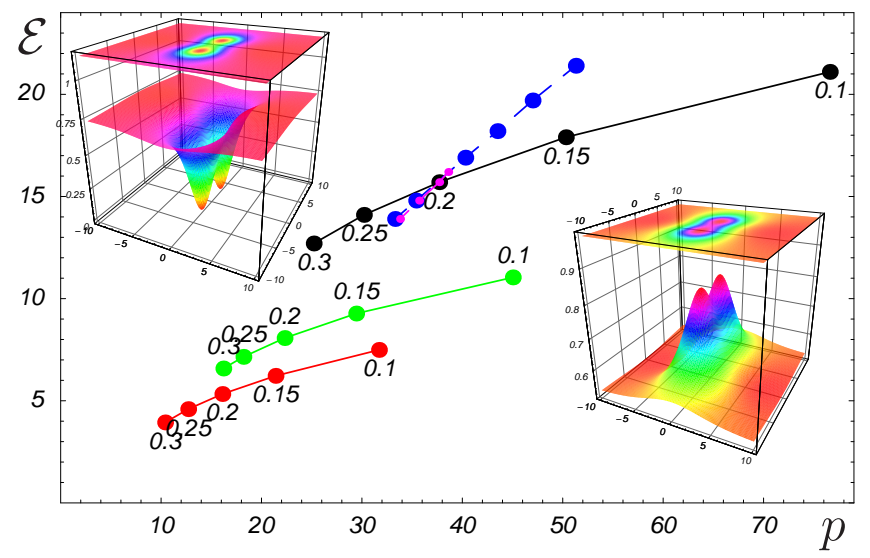

$z$-direction. For simplicity in this section we let $\alpha_{1}=\alpha_{2}=\alpha$ and $\gamma=1$. The amplitudes of the ground state satisfy the following ordinary differential equations:

$$
\begin{aligned}
& R_{1}^{\prime \prime}+\frac{R_{1}^{\prime}}{s}-\frac{R_{1}}{s^{2}}+\left(1-R_{1}^{2}-\alpha R_{2}^{2}\right) R_{1}=0 \\
& R_{2}^{\prime \prime}+\frac{R_{2}^{\prime}}{s}+\left(1-\alpha R_{1}^{2}-R_{2}^{2}-\Lambda^{2}\right) R_{2}=0
\end{aligned}
$$

The solutions of Eqs. 42 are shown graphically on FIG.8 for a variety of values of $\alpha$ and $\Lambda^{2}$. The asymptotic behaviour at infinity is easily obtained 


\section{Two-Component Bose Condensates}

Fig. 8. (colour online) The solutions of Eqs. (42) for the amplitudes of the ground state consisting of a straight line vortex in the first component. The inserts show the values of the parameters used in the order of the plots (from the top to the bottom).
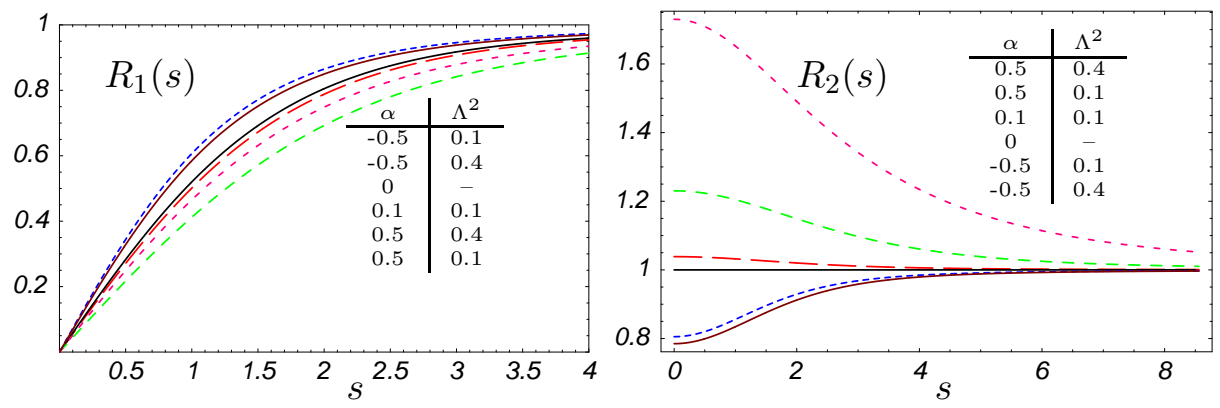

as

$$
\begin{aligned}
& R_{1} \sim \psi_{1 \infty}-\frac{1}{2 \psi_{1 \infty} s^{2}} \\
& R_{2} \sim \psi_{2 \infty} \pm K_{0}\left(2 \psi_{2 \infty}^{2} s\right) \sim \psi_{2 \infty} \pm \exp \left(-2 \psi_{2 \infty}^{2} s\right)
\end{aligned}
$$

When ansatz (41) is used in Eqs. (24) the equations on $\chi_{i}$ become

$$
\begin{aligned}
& 2 \mathrm{i} U \frac{\partial \chi_{1}}{\partial z}=\frac{1}{r} \frac{\partial}{\partial r}\left[r \frac{\partial \chi_{1}}{\partial r}\right]+\frac{\partial^{2} \chi_{1}}{\partial z^{2}}-\frac{\chi_{1}}{r^{2}} \\
& \quad+\left(1-\left|R_{1}+\chi_{1}\right|^{2}-\alpha\left|R_{2}+\chi_{2}\right|^{2}\right)\left(R_{1}+\chi_{1}\right)-\left(1-R_{1}^{2}-\alpha R_{2}^{2}\right) R_{1} \\
& 2 \mathrm{i} U \frac{\partial \chi_{2}}{\partial z}=\frac{1}{r} \frac{\partial}{\partial r}\left[r \frac{\partial \chi_{2}}{\partial r}\right]+\frac{\partial^{2} \chi_{2}}{\partial z^{2}} \\
& \quad+\left(1-\alpha\left|R_{1}+\chi_{1}\right|^{2}-\left|R_{2}+\chi_{2}\right|^{2}-\Lambda^{2}\right)\left(R_{2}+\chi_{2}\right) \\
& \quad-\left(1-\alpha R_{1}^{2}-R_{2}^{2}-\Lambda^{2}\right) R_{2} .
\end{aligned}
$$

These equations were solved by Newton-Raphson iterations to give the families of the localized disturbances that move along the vortex line with a constant velocity and preserving their form. FIG. 9 gives the dispersion curves of these families for $\alpha=0.1$ and $\Lambda=0.1$. FIG. 10 shows the density isosurfaces of various solitary waves.

\section{CONCLUSIONS}

In summary, we considered the coupled GP (NLS) equation as a model of two-component Bose gas. We showed that this model exhibits a conden- 


\section{Natalia G. Berloff and Chen Yin}

Fig. 9. (Colour online) The dispersion curves of three families of the axisymmetric solitary wave solutions moving along a vortex line as solutions of Eq. (24) with $\gamma=1, \alpha=0.1$ and $\Lambda^{2}=0.1$. The energy $\widehat{\mathcal{E}}$ is calculated by subtracting the energy of the vortex from the energy of the complex. The numbers next to the dots give the velocity of the solitary wave solution. The top (black) branch corresponds to VR-VR and VR-RP complexes. The middle (green) branch shows VR-SW complexes and the bottom (red) branch is the dispersion curve of SW-VR and SW-RP complexes.

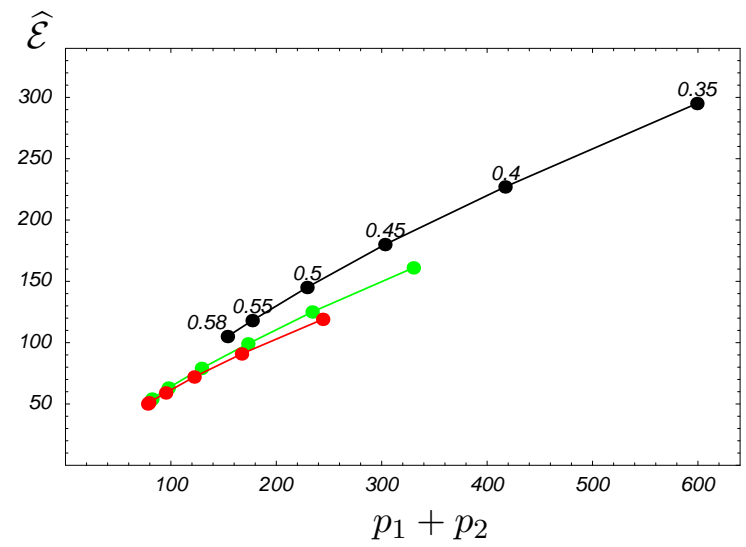

Fig. 10. (Colour online) The density isosurfaces of various solitary wave solutions that propagate along the vortex line. The isosurfaces shown are $\rho_{1}=\frac{1}{5} \psi_{1 \infty}^{2}$ (green), $\rho_{2}=\frac{1}{5} \psi_{2 \infty}^{2}$ (red), $\rho_{2}>\psi_{2 \infty}^{2}$ (blue) and $\rho_{1}>\psi_{1 \infty}^{2}$ (gray).
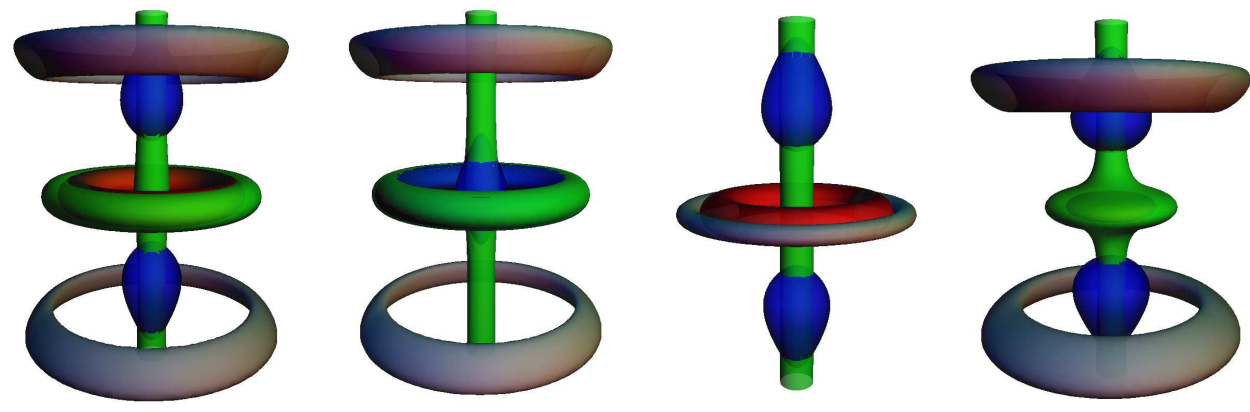


\section{Two-Component Bose Condensates}

sation process in 3D. Starting with a random stochastic initial condition we derived the kinetic equation that describes the initial time evolution of the system. The system evolves into the state of thermodynamical equilibrium with condensates in either both components or in a single component only. The addition of the second gas increased the number of condensed particles in the first gas. The condensates are formed with a tangle of topological defects. During the decay of the tangle the large wavelength part of the system evolves into solitary wave structures. We found the families of these structures in uniform 3D and $2 \mathrm{D}$ condensates as well as the families of the disturbances moving along the topological defects. The process of the classical wave condensation with the formation of solitary wave structures can be viewed as an analog of the Kibble-Zurich transition.

\section{ACKNOWLEDGEMENTS}

NGB thanks Boris Svistunov for a useful discussion and EPSRC(UK) for financial support. CY is grateful to PPARC for his studentship.

\section{REFERENCES}

1. C.J. Myatt et al., Phys. Rev. Lett. 78, 586 (1997); D.S.Hall et al., Phys. Rev. Lett. 81, 1539 (1998); D.M. Stamper-Kurn et al., Phys. Rev. Lett. 80, 2027 (1998) and J. Stenger et al., Nature 396, 345 (1998).

2. G.Modugno et al., Phys. Rev. Lett. 89, 190404 (2002).

3. G. Volovik, "The Universe in a Helium Droplet," Clarendon Press, Oxford (2003).

4. E. Levich and V. Yakhot, J. Phys. A: Math. Gen. 11, 2237 (1978) and Yu. Kagan and B.V. Svistunov, Phys. Rev. Lett. 79, 3331 (1997).

5. V.E.Zakharov, S.L. Musher, and A.M.Rubenchik, Phys. Rep. 129, 285 (1985) and S. Dyachenko, A.C. Newell, A. Pushkarev and V.E.Zakharov, Physica D 57, 96 (1992).

6. B.V. Svistunov, J. Moscow Phys. Soc. 1, 373 (1991).

7. Yu. Kagan, B.V. Svistunov, and G.V. Shlyapnikov, Zh. Eksp. Teor. Fiz. 101, 528 (1992) [Sov. Phys. JETP 75, 387 (1992)]; Yu. Kagan and B.V. Svistunov, Zh. Eksp. Theor. Fiz. 105, 353 (1994) [Sov. Phys. JETP 78, 187 (1994)].

8. N.G.Berloff and B.V. Svistunov, Phys. Rev. A 66, 013603 (2002).

9. M.J.Davis, S.A. Morgan, and K. Burnett, Phys. Rev. Lett 87, 160402 (2001) and Phys. Rev. A 66, 053618 (2002).

10. S. Nazarenko and M. Onorato, arXiv:nlin.CD/0507051, (2005)

11. M.Kobayashi and M.Tsubota, Phys. Rev. Lett. 94, 065302 (2005).

12. C.J. Pethick and H.Smith, "Bose-Einstein Condensation in Dilute Gases", Cambridge University Press (2002). 


\section{Natalia G. Berloff and Chen Yin}

13. C. Connaughton et al, Phys. Rev. Lett. 95, 26901 (2005).

14. N.G. Berloff, Phys. Rev. Lett. 94 , 120401 (2005).

15. N.G. Berloff, J. Phys. A: Math. Gen. 37, 1617 (2004).

16. T.W.B. Kibble, J. Phys. A 9, 1387 (1976) and W. H. Zurek, Nature 317, 505 (1985).

17. P. Tommasini et al, Phys. Rev. A 67, 023606 (2003).

18. P. Öhberg and L. Santos, Phys. Rev. Lett. 86, 2918 (2001); Th. Busch and J.R. Anglin, Phys. Rev. Lett. 87, 010401 (2001) and P.G. Kevrekidis et al., Eur. Phys. J. D 28, 181 (2004).

19. S. Coen and M. Haelterman, Phys. Rev. Lett. 87, 140401 (2001).

20. J. Ruostekoski and J.R. Anglin, Phys. Rev. Lett. 86, 3934 (2001); C.M. Savage and J. Ruostekoski, Phys. Rev. Lett. 91, 010403 (2003) and R.A. Battye, N.R. Cooper and P.M. Sutcliffe, Phys. Rev. Lett. 88, 080401 (2002).

21. C.A. Jones and P.H. Roberts, J. Phys. A 152599 (1982) and C.A.Jones, S.J. Putterman, and P.H. Roberts, J. Phys. A: Math. Gen. 192991 (1986)

22. N.G. Berloff and P.H. Roberts, J. Phys. A:Math. Gen. 37, 11333 (2004).

23. N.G. Berloff, Phys. Rev. Lett. 94, 010403 (2005). 